\title{
Attitudes on Health Promotion among Medical Students and Implications for Future Practice
}

\author{
T.I.Pathirana ${ }^{1}$, S. Larmar ${ }^{2}$
}

\begin{abstract}
A lack of positive attitude towards health promotion is identified as a major barrier encountered by physicians in delivering effective health promotion to patients and the wider community. As a key component of physician training, medical students would benefit from regular 'attitudinal' screening and professional guidance to cultivate both positive attitudes and corresponding professional responses towards health promotion. This review willdelineate the multiple and diverse predictors that influence attitudes towards health promotion over time. The paper also provides recommendations to generate better attitudes inmedical practitioners in training. Finally, the review advocates for the ongoing facilitation of longitudinal follow up studies investigating trends in the formation of positive attitudes towards health promotion in medical students to establish effective evidence based strategies to further enhance health promoting attitudes and behaviors.
\end{abstract}

Key words: Health promotion, Attitudes, Medical students, Undergraduate medical education,

\section{Introduction}

Since its inception in the Ottawa Charter in 1986, health promotion has become a widely accepted concept worldwide (1). Nevertheless, thereis ample evidence that its application is still deficient in many key areas of disease prevention (2). For example, it is well documented that many doctors fail to counsel their patients appropriately on healthy lifestyle behaviors in spite of its proven health gains (3). Lack of positive attitude, self-confidence and skills to convey health promotion information tailored to individual or community needs are recognized as major barriers in health promotion by physicians (4). This emphasizes the role of undergraduate medical education in intervening to ensure that positive attitudes towards health promotion are developed as a part of the process of physician training $(3,5,6)$. Such attitudes serve to influence foundational learning outcomes in health promotion culminating in the enhancement of future practice (5). This review attempts to describe and critically analyze the available literature focusing on medical students' attitudes towards health promotion, including predictors of positive attitude development and implications for future practice.

\section{Methodology}

An electronic search of the following databases was performed which included Pubmed, Science Direct, Proquest and Scopus. Boolean logic was utilized to combine a collection of key search terms including health promotion, attitude, medical student, medical education and behavior. Peer-reviewed articles published within the last 10 years (October 2003-October 2013) were selected in addition to key texts and information derived from the World health Organization's official website. These sources of information were reviewed, critically evaluated and narratively summarized utilizing the main themes identified as an overarching framework to guide the review. Most studies had employed selfreported surveys which included the Prevention

1. Masters student in Public Health Griffith University Australia

2. Senior Lecturer, School of Human Services and Social work, Griffith University, Australia

Correspondence: thanindu@gmail.com 
Attitude Scale (PAS)(5) and Attitudes to Social Issues in Medicine (ATSIM) scale (7) to measure the attitudes of medical students on health promotion and prevention.

\section{Mixed attitudes in medical students on health promotion}

While early studies facilitated in the 1990s show relatively consistent positive attitudes among medical students towards the basic concepts of health promotion $(8,9)$, more recent evidence tends to identify mixed attitudes. Studies involving medical students in California (5) and Australia (3) demonstrated a varying range in the positive attitudes toward health promotion and prevention. The Californian study facilitated by Bellas et al was carried out as part of a curriculum evaluation survey with the participation of 599 first year medical students from five medical schools. Of the participants included in the sample, $98 \%$ agreed on the cost effectiveness of preventative medicine while only $63 \%$ agreed on health promotion as the major role of a primary care physician (5). The study conducted by Gnanendran et al focused on exercise counseling in the practice of preventative medicine and involved 131 medical students from the Australian National University. This study revealed that $66 \%$ of participants agreed on prevention being as equally important as treatment(3).

In a study conducted in Germany by Klement et al, 220 fifth year medical students were recruited to explore participant perceptions of health promotion and prevention(10). The findings of this study reported that the relative importance assigned by the students towards several key areas in health promotion and preventive medicine such as counseling on environmental and genetic risk factors were found to be markedly low.

\section{Predictors of attitudes towards health promotion}

Several key predictors were highlighted in the literature that may ultimately determine and influence attitudes towards health promotion and their observed differences. The majority of past studies $(11,12)$ as well as more recent investigations (10) identified female gender as a favorable predictor of positive attitude towards health promotion with ratings on its importance being significantly higher compared to males. For instance in the study by Klement et al, $51 \%$ of the females as compared to $33 \%$ of males rated population based preventative measures to be important or very important while $46 \%$ of females as compared to $34 \%$ of males rated risk communication to be important or very important. Furthermore, $73 \%$ of females acknowledged the importance of preventive medicine and health promotion within their personal medical profession compared to only $57 \%$ of males $(10)$.

The study conducted by Bellas et al involving Californian medical students revealed that a future preference for a career in the specialty of preventive medicine or primary care also had a positive influence on attitudes towards health promotion (5). Further, the study identified that personal beliefs regarding social determinants of health were also strong predictors of positive attitude which is consistent with the findings of the study conducted by Klement et al in Germany (10). Interestingly this study also revealed that Democratic Party preference was identified as a significant positive influential factor that may highlight the socioeconomic and political contexts within which health promotion policies are shaped and implemented (5).

Previous experiences and practices related to healthy living have also been shown to impact onattitudes. For example, in the Australian study by Gnanendran etal involving both medical 
students and physicians, it was interesting to note that participants who had led active lives in their youth had more favorable attitudes towards health promotion counseling with regard to physical activity (3).

\section{A change of attitudes in medical schools}

Previous trends appear to indicate that attitudes towards health promotion for medical students and physicians were 'brought to medical school' rather than inculcated during training (13). In contrast, a more recent study facilitated in Colombia by Duperly et al involving661 first and fifth year medical students revealed thatattitudes evolve during the period of medical student undergraduate training (14). An Australian study conducted by Price et al compared 385 medical students in their first, fifth and sixth (final) years and revealed that the greatest attitudinal change occurred up to fifth year with insignificant change identified thereafter(15). Furthermore, Ewan (16) followed up a cohort of Australian medical students from their first year of study in to their senior years with the research identifying that as participants progressed through a medical training course they were less likely to appreciate the social determinates of healthessential to successful health promotion in community populations.

\section{Implications}

As attitudes of medical students on health promotion were found to be correlated with healthy life style behaviors as well as health promotion practices (3), it is worthwhile discussing this trend further. In the study byGnanendran et al (3), one third of the participating students admitted that they failed to engage themselves in sufficient physical activities. This in turn was linked with the attitude that exercise was not a crucial component of their daily lives. Similar trends were described in the study by Duperly et al
(14), where medical students smoked more and consumed a less healthy diet when compared to the general population in the same age group. Interestingly, participants were more inclined towards these unhealthy behaviors by the time they had reached the final year of medical study compared to students in first year. This may be partly due to the highly stressful and time demanding nature of academic life as students progressed through the degree program (17).Consequently, such unhealthy behavior patterns were correlated with less favorable attitudes towards health promotion (18). Conversely, negative attitudes towards health promotion have been shown to hinder the health promotion counseling delivered by a health professional(19).

\section{Interventions for improving attitudes on health promotion}

Identified interventions targetingmedical students with a focus on promoting positive attitudes towards healthy behaviors have strongly encouraged student practitioners in training to offer better health promotion advice in comparison to students who were not exposed to such interventions. Interventions have included improving accessibility to extracurricular activities such as physical activity sessions including yoga, team sports or taking part in other organized exercise classes within the medical school context $(18,20)$

Several studies have stressed the significance of revising traditional preventive medicine programs as a means to developing a more integrated curriculum approach $(21,22)$. Integrated curriculum models give emphasis to key principles in health promotion and are taught interactively across training programs within themainstream curriculum by clinicians who are regarded by students as role models (5). Such role models would serve to maintain student interest. 
in, and improve student attitudes towards, health promotion if various personal and socio political values of medical students were taken into account.

Given that personal beliefs (9) and values (3) seem to play a crucial role in determining ongoing positive attitudes towards health promotion, it may also be prudent to employ rigorous screening measures to evaluate the attitudes and values of applicants on admission to medical schools (23).

The broad spectrum of cognitive, affective and behavioral dimensions that must be considered when defining attitudes towards health promotion, renders the task of accurately measuring such attitudes in regular community samples as highly complex (24). Utilizing selfreported questionnaires to assess such attitudes creates a further limitation as measures of this kind can elicit a subjective evaluation favoring the tendency to report attitudes which are positively framed but disingenuous (3).

\section{Limitations and future recommendations}

Medical students' positive attitudes towards health promotion are crucial to the role of consulting physicians working within regular community populations. Thus the multiple and diverse predictors influencing these attitudes should be further investigated in different contexts and cogent intervention approaches developed to promote positive attitudes towards health promotion. Given the dearth of studies documenting medical students' attitudes towards health promotion during training, as well as the limited findings to highlight the complex associations influencing such attitudinal changes, longitudinal follow up and evaluation studies of medical students in both developing and developed countries are necessary in order to establish effective evidence based strategies which would enhance their health promoting attitudes and behaviors. Such studies would help determine the efficacy of specific interventions to strengthen attitudes towards health promotion as well as define the best educational approaches that would encourage positive attitudes towards health promoting activities. These strategies would in turn have a positive outcome on the health promotion activities physicians would implement in their practice leading to enhanced patient engagement and broader community health outcomes.

\section{Conclusions}

This review focused on the significance of medical student attitudes towards health promotion and the implications of positive attitudes on health promotion practices. A range of studies was identified that delineated the significance of positive health promoting attitudes, their predictors and potential interventions to encourage positive attitudinal positions concerning health promotion in regular community settings. The paper highlighted the significance of developing positive attitudes towards health promotion and prevention and the implications of such attitudes in contributing to the wellbeing of patients and physicians. The review also identified the dearth of research in the area and the implications of limited studies exploring this phenomenon given that multiple and diverse predictors concerning the development of positive attitudes in health promotion for practitioners in training exist within a range of contexts, including developing nations. Finally, future research agendas focusing on effective educational and behavioral interventions were recommended that would serve to redress the significant research gap as a means of encouraging future physicians' positive attitudes towards health promotion and prevention. 


\section{Acknowledgement}

Thanya Pathirana was supported by the Fogarty International Centre, National Institutes of Health, under Award Number: D43TW008332 (ASCEND Research Network). The contents of this publication is solely the responsibility of the authors and does not necessarily represent the official views of the National Institutes of Health or the ASCEND Research Network.

\section{References}

1. World Health Organization. The Ottawa Charter for Health Promotion: First International Conference on Health Promotion, Ottawa, 21 November 1986 Geneva: World Health Organization; 1986. Available from: http://www.who.int/ healthpromotion/conferences/previous/otta wa/en/print.html.

2. World Health Organization. Assessing national capacity for the prevention and control of noncommunicable diseases: report of the 2010 global survey. 2012.

3. Gnanendran A, Pyne DB, Fallon KE, Fricker PA. Attitudes of medical students, clinicians and sports scientists towards exercise counselling. 2011.

4. Frank E, Kunovichfrieze T. Physicians' Prevention Counseling Behaviors: Current Status and Future Directions. Preventive Medicine. 1995;24(6):543-5.

5. Bellas PA, Asch SM, Wilkes M. What students bring to medical school: Attitudes toward health promotion and prevention. American Journal of Preventive Medicine. 2000;18(3):242-8.

6. Williford HN, Barfield BR, Lazenby RB, Olson MS. A survey of physicians' attitudes and practices related to exercise promotion. Prev Med. 1992;21(5):630-6.

7. Ewan CE. Attitudes to social issues in medicine: a comparison of first-year medical students with first-year students in nonmedical faculties. Medical education. 1987;21(1):25-31.
8. Meakin RP, Lloyd MH. Disease prevention and health promotion: a study of medical students and teachers. Medical education. 1996;30(2):97-104.

9. Greenland P, Derby CA. Medical students' improved attitudes toward prevention of cardiovascular diseases from entry to graduation. Preventive Cardiology Academic Cooperative Research Group ofthe National Heart, Lung, and Blood Institute. Am J Prev Med. 1992;8(1):53-7.

10. Klement A, Bretschneider K, Lautenschläger C, Stang A, Herrmann M, Haerting J. Prevention and health promotion in undergraduate medical education: Preferences, attitudes and previous knowledge of medical students - a crosssectional study. GMS Zeitschrift für medizinische A u sbild ung. 2011;28(1):Doc17.

11. Bertakis KD, Helms LJ, Callahan EJ, Azari $\mathrm{R}$, Robbins JA. The influence of gender on physician practice style. Medical care. 1995;33(4):407-16.

12. Lurie N, Slater J, McGovern P, Ekstrum J, Quam L, Margolis K. Preventive Care for Women -- Does the Sex of the Physician Matter? The New England Journal of Medicine. 1993;329(7):478-82.

13. Konen JC, Fromm BS. Changes in personal health behaviors of medical students. Medical teacher. 1992;14(4):321-5.

14. Duperly J, Lobelo F, Segura C, Sarmiento F, Herrera D, Sarmiento OL, et al. The association between Colombian medical students' healthy personal habits and a positive attitude toward preventive counseling: cross-sectional analyses. BMC public health. 2009;9(1):218-.

15. Price J, Price D, Williams G, Hoffenberg R. Changes in medical student attitudes as they progress through a medical course. Journal of medical ethics. 1998;24(2):110-7. 
16. Ewan CE. Social issues in medicine: a follow-up comparison of senior-year medical students' attitudes with contemporaries in non-medical faculties. Medical education. 1988;22(5):375-80.

17. Dahlin M, Joneborg N, Runeson B. Stress and depression among medical students: a cross-sectional study. Medical education. 2005;39(6):594-604.

18. Frank E, Tong E, Lobelo F, Carrera J, Duperly J. Physical activity levels and counseling practices of U.S. medical students. Medicine and science in sports and exercise. 2008;40(3):413-21.

19. Frank E, Breyan J, Elon L. Physician Disclosure of Healthy Personal Behaviors Improves Credibility and Ability to Motivate. Archives of Family Medicine. 2000;9(3):287-90.

20. Gupta K, Fan L. Doctors: fighting fit or couch potatoes? British journal of sports medicine. 2009;43(2):153-4.
21. Litaker D, Cebul RD, Masters S, Nosek T, Haynie R, Smith CK. Disease prevention and health promotion in medical education: reflections from an academic health center. Academic medicine : journal of the Association of American Medical Colleges. 2004;79(7):690-7.

22. Sutphen SM, Cibula DA, Morrow CB, Epling JW, Novick LF. Evaluation of a preventive medicine curriculum: incorporating a case-based approach. American journal of preventive medicine. 2003;24(4 Suppl):90-4.

23. Linzer M, Slavin T, Mutha S, Takayama JI, Branda L, VanEyck S, et al. Admission, recruitment, and retention: finding and keeping the generalist-oriented student. SGIM Task Force on Career Choice in Primary Care and Internal Medicine. Journal of general internal medicine. 1994;9(4 Suppl 1):S14-23.

24. Downie RS, Tannahill C, Tannahill A. Health promotion: models and values. Oxford: Oxford University Press; 1996. 